\title{
La justificación: condición de calidad del programa de psicología de CECAR
}

\section{Justification: quality condition for CECAR's psychology program}

\author{
María José Martínez Luna \\ Corporación Universitaria del Caribe - CECAR, Sincelejo, Colombia
}

\section{Resumen}

Introducción: La educación es un derecho fundamental de todos y cada uno de los ciudadanos, independientemente de su nivel socioeconómico; sin embargo, para que esta repercuta en el desarrollo integral de los educandos, debe cumplir con ciertos requisitos de eficacia acordes con las necesidades de la sociedad y las capacidades intelectuales de los individuos. En este sentido, se han establecido condiciones mínimas de calidad para el mejoramiento de la educación superior en Colombia, de tal manera que las instituciones y centros educativos alcancen en realidad dicha meta. Objetivo: Analizar la justificación del programa de Psicología de la Corporación Universitaria del Caribe-CECAR. Método: El estudio es de carácter cualitativo y consiste en una revisión documental. Se examina la justificación como condición de alta calidad de un programa de Psicología, incluida en el documento maestro del Registro Calificado. Resultados: El análisis de los tres criterios básicos de la justificación del programa de psicología atiende a las características de la condición de calidad n02 justificación. Discusión y Conclusiones: El programa de Psicología de la Corporación Universitaria del Caribe-CECAR cumple con las características básicas de la condición de calidad n02 justificación, y por esta razón obtuvo la acreditación de alta calidad de educación superior, constituyéndose en la única corporación de la ciudad de Sincelejo que cuenta con el programa de Psicología acreditado en alta calidad.

Palabras clave: Educación, educación superior, calidad educativa.

\begin{abstract}
Introduction: Education is a fundamental right for each and every citizen regardless of socioeconomic stratum; however, In order to impact on integral development of learners, education must meet certain requirements of effectiveness according to the society needs and individuals intellectual abilities. That is why, minimum quality conditions for the improvement of higher education in Colombia have been suggested, in order to be sure that not only educational institutions but also centers are achieving those proposed goals. Objective: This paper aims to analyze the justification of the Psychology program of the Caribbean University Corporation CECAR. Method: A qualitative study, with a type of documentary review investigation is conducted. Justification is examined as a high quality condition of the psychology program, included in the Qualified Record master document. Results: the analysis of the three basic criteria of this justification for the psychology program meets the characteristics of the quality condition No. 2 justification. Discussion y Conclusion: Since CECAR's psychology program (Corporaciòn Universitaria del Caribe), meets the basic characteristics of the quality condition No. 2 justification, being this one the only corporation in Sincelejo, has been credited as a high quality psychology program.

Keywords: Education, higher education, educational quality.
\end{abstract}

Open Access:

ISSN: 0124-2121 E-ISSN: 2665-2420

ARTÍCULO RSULTADO DE INVESTIGACIÓN

Copyright (c) 2020

By Educación y Humanismo

Editor:

Patricia Martínez Barrios Universidad Simón Bolivar

\author{
Correspondencia: \\ María Martínez \\ maría.martinez|@cecar.edu.co
}

Recibido: 19-05-2019

Aceptado: 05-09-2019

En línea desde: 02-12-2019

10.17081/eduhum 22

38.3455 


\section{Introducción}

Desde la perspectiva legal de los derechos humanos, la educación es un derecho fundamental para todas las personas (Tomasevski, 2004). También está claro que la formación personal y social depende de la eficacia de las instituciones y centros que prestan el servicio y su necesidad universal de cobertura (Beltrán-Villamizar, Matínez-Fuentes \& Vargas-Beltrán, 2015). Por otra parte, se puede decir que el régimen formativo en Colombia es de carácter acumulativo, ya que atenúa los conocimientos, habilidades y capacidades, pertinencia y transformación (Celis, Jiménez \& Jaramillo, 2012). Como propone Rama (1970), la calidad del sistema debe evaluarse de forma que resulte provechoso en el contexto donde se encuentra inmerso, pues, el acto educativo se refleja en los aportes que le proporciona a la estructura socioeconómica y en la satisfacción de las necesidades de la comunidad. Según Roa (2003), además, la evaluación debe comprender muchas dimensiones y panoramas según el ámbito. En este orden de ideas, Tobón (2006) afirma que la evaluación de la educación debe propiciar estrategias que favorezcan y aseguren los ejes centrales de dicha educación, gestionando eficacia para las didácticas del aprender. Y, en efecto, la educación se entiende como un proceso que permite que las personas crezcan y avancen en su vida profesional y personal al mismo tiempo. Los procesos educacionales implican impartir conocimientos a un grupo de individuos acerca de una temática o área en especial, que requiere de destrezas y adecuaciones acordes con la preparación. Por eso, necesitan ciertos requerimientos básicos, como espacios adecuados para estimular y propiciarlo; sujetos con una formación apropiada de la disciplina y, sobre todo, con alineación pedagógica, desde metodologías de instrucción hasta tácticas que permitan facilitarla. Según Burton (1991), el organismo educativo, a diferencia de otros en nuestra sociedad, se caracteriza porque su estructura está basada en el conocimiento, la ciencia y enseñanza-aprendizaje; es un sistema social que por excelencia avanza notoriamente con la evolución y los cambios del día a día. Concordando con lo anterior, Peralta (2009) plantea que la organización educativa de Colombia posibilita el ejercicio del impulso global a partir de una perspectiva humanística que promueve el desarrollo de la comunidad.

Por todo lo anterior, la educación es un eje social que debe articularse con las necesidades del contexto donde se desarrolla, a fin de que posibilite un modelo económico y sociopolítico ajustable a la sociedad; por lo tanto, la deficiencia de esta constituye un grave retroceso (Colbert, 1999). Y es que la educación siempre es una respuesta a los fenómenos de una comunidad y cultura en particular, y en ella se reconoce la calidad de un sistema educativo (Aguerrondo, 1993). Precisamente allí radica la importancia de velar por unos procesos de excelencia; con calidad, con propósitos que a su vez formen y estimulen el conocimiento en general, propiciando la equidad y asumiendo decisiones que construyan cambios reales (Valencia, 2008). En este sentido, América Latina se encuentra en el tercer puesto del índice de progreso en materia de conocimiento (Brunner, 2010), estando apenas por encima del Sur de Asia y África, un nivel relativamente bajo teniendo en cuenta los escalafones de Europa; y en este escenario, Colombia aparece ubicada en el noveno puesto 
del índice de conocimiento seguido por Venezuela y República Dominicana.

Para analizar la educación del país, se debe revisar primero el contexto de esta, contemplar, por ejemplo, los avances, la globalización, el capitalismo, el orden político, las proyecciones sociales, las comunicaciones, factores determinantes en la autonomía del aprendizaje (Salas, 2005). En la actualidad, la educación superior colombiana es objeto de muchos cambios en su estructura y organización, pero Viloria (2006) sostiene que, por muchos cambios que se pretendan, la educación superior en el Caribe colombiano está llena de limitaciones y problemas, como muestran las estadísticas de las pruebas ECAES; la cobertura que, aunque se ha tratado de ampliar, todavía no se asemeja a la media nacional; dificultades en el acceso a los créditos educativos y escasa relación entre las ofertas de pregrado y posgrado; y el estado del mercado actual. Ciertamente, en Colombia existen universidades de gran prestigio que cuentan con un estatus de calidad impresionante; pero al mismo tiempo, existen instituciones de educación superior, públicas y privadas, que están en camino de convertirse en universidades, a fin de obtener cierto nivel de crecimiento. Sin embargo, el reconocimiento de una universidad realmente se basa en la calidad educativa, razón por la cual los resultados de los procesos de educación se evidencian en el tipo de profesionales que egresan y por el impacto que genera ese programa de formación en la comunidad en general. En este sentido, muchas instituciones que ofrecen educación profesional, no cumplen con todos los requisitos para brindar una formación completa.

Melo-Becerra, Ramos-Forero y Hernández-Santamaría (2017) afirman que la educación superior en Colombia es de una calidad marcadamente diversa, pues, así como existen instituciones con un gran reconocimiento, también existen otras de baja calidad. A esto se suma la falta de conexión entre el sector productivo y la formación profesional que se brinda, lo que limita de cierta forma el desarrollo económico del país. Desde la perspectiva de estos autores, la importancia de un programa académico dentro de un contexto en específico debe ser fundamental, en el sentido de que ella debe satisfacer las necesidades de este contexto para que adquiera un grado de pertinencia aceptable tanto en el ámbito académico como en el ámbito socioeconómico. En esta misma línea, Garbanzo (2007) plantea que la educación superior debe mantener relación con el sector público, de modo que haya un adecuado costo-beneficio social; por lo tanto, se debe prestar gran atención a los indicadores que guían los procesos académicos y así determinar las mejores opciones y alternativas entre las instituciones de educación superior y la sociedad.

Existe un órgano que controla y vigila a las instituciones y centros que ofrecen el servicio de educación superior: el Sistema de Aseguramiento de la calidad de la Educación Superior en Colombia, manejado desde el Plan Sectorial 2006-2010. Aunque este sistema de aseguramiento de la calidad no desarrolla a fondo todos los principios y fines planteados, se presenta como detallista y minucioso ante las acciones que se deben llevar a cabo (Miñana \& Rodríguez, 2011). Asimismo, las leyes que soportan el proceso educativo, como la Ley 115 de 1994 o Ley General de Educación, resaltan todos los aspectos que rigen la educación en Colombia, como un proceso de instrucción y crecimiento constante que 
fortalece la dimensión personal, social, cultural e integrativa (Ley 115, 1994). Tal como lo explica Naranjo (2009), se deben establecer consideraciones finales de motivación hacia la educación, es decir, que los mismos regímenes que la sobrellevan deben encargarse de transmitir los beneficios de acceder a esta. De hecho, en la Ley 30 de 1992 Servicio Público de Educación Superior se establecen las disposiciones para la estructuración de la educación superior como parte de la formación académico-profesional (Consejo Nacional de Acreditación CNA, 1992). Según Gómez y Celis (2009), un número significativo de personas de los estratos más bajos del país no pueden acceder a la educación superior. Ello ha generado propuestas de políticas sociales y un sistema de equidad de la educación universitaria, con el fin de que los jóvenes de todos los estratos puedan entrar a la universidad.

El Decreto 2566 de 2003 instituye las condiciones mínimas de calidad para la educación superior, acorde con la Constitución Política y las leyes antes mencionadas y el Decreto 2904 de 1994, donde se encuentra el reglamento de los artículos 53 y 54 de la Ley 30 de 1992, para la creación del Sistema Nacional de Acreditación-SNA y el Consejo Nacional de Acreditación-CNA (Ministerio de Justicia, 2009). A pesar de todas estas acciones y normas, siempre ha habido limitaciones que afectan su cumplimiento en los procesos educativos; dejando a un lado el sistema de control y vigilancia, cuyo objetivo fundamental es velar por la calidad educativa, pero desde el que, al mismo tiempo, se malinterpreta la autonomía de las universidades y se asume, en cambio, un "libertinaje educativo", propiciando un nivel de calidad inadecuado para los programas que ofrecen (Giraldo, Abad \& Díaz, 2007).

El departamento de Sucre, en Colombia, se caracteriza por tener un sistema económico basado en la ganadería, el comercio y la agricultura, lo que permite inferir que su modelo económico aún se encuentra basado en la materia prima. A pesar de ello, se puede ver un crecimiento en el mercado con la inmersión de un sinnúmero de empresas de diversos orígenes, que contribuyen a la expansión de la economía. No obstante, Sucre ha sido uno de los departamentos más afectados por los fenómenos de la violencia, el desplazamiento, el narcotráfico, tal como lo comprueba el Plan de Desarrollo de Sucre 2016-2019, donde se establece que el $59.40 \%$ de la población está caracterizada como víctima. Lo anterior ha provocado el traslado de personas de muchos pueblos hasta la ciudad, principalmente a Sincelejo, lo que aumenta la población y disminuye las oportunidades de empleo (Secretaria de Planeación Municipal, 2016 - 2019). Teniendo en cuenta esto, Bonilla (2011) refiere que la educación es un factor primordial en la expansión del sector económico, asumiendo que esta impacte positivamente en el mercado.

Por otra parte, en la ciudad de Sincelejo existen diversas instituciones de educación superior que ofrecen una amplia gama de programas universitarios, como la Universidad de Sucre, la Corporación Universitaria Antonio José de Sucre y la Corporación Universitaria del Caribe-CECAR, entre otras. Estas dos últimas instituciones son "corporaciones", lo cual significa que aún no han llegado a certificarse como universidades o como instituciones de alta calidad. La Corporación Universitaria del Caribe-CECAR está justamente en ese proceso, 
que inició con la obtención de la acreditación del programa de Psicología. Cabe aclarar que CECAR no es el único centro educativo que ofrece estudios de Psicología en Sincelejo, sino también la Corporación Universitaria Antonio José de Sucre. Con unos antecedentes de violencia, una población en constante crecimiento, un modelo económico que no satisface las necesidades de la comunidad y con unas instituciones de educación superior de las que egresan un sinnúmero de profesionales cada año y que en el caso de la oferta de Psicología se brinda por dos centros educativos, la ciudad de Sincelejo evidencia una brecha competitiva que se debería aprovechar y motivar, así, el progreso social. Al respecto, expresa Restrepo (2003) que los fenómenos sociales siempre van a existir, pero el surgimiento de disciplinas como la psicología, perteneciente a las ciencias sociales, constituyen un paradigma de transformación y replanteamiento de la sociedad y sus enfoques vivenciales.

Es así como la pertinencia de un programa de pregrado en Psicología radica en la necesidad de los procesos formativos en este campo que exige el mundo actual y en la excelencia del desempeño académico, social y laboral (Herrera, Restrepo, Uribe \& López, 2009). En Colombia existen 138 instituciones de educación superior que ofrecen el programa de pregrado de psicología. No obstante, solo 43 se encuentran acreditadas con alta calidad (ASCOFAPSI, 2012), es decir, que ni siquiera el 50\% de los centros que lo imparten cumplen con los criterios mínimos planteados para la educación de alta calidad. Es prioritario establecer un abordaje psicológico basado en el contexto del Caribe colombiano, y las universidades que ofrecen el programa en la región deben incluir esta prioridad en la formación de los profesionales en el área de la salud mental (Silva \& Acevedo, 2011). Desde esta perspectiva, es sumamente necesario indagar las condiciones en las que se encuentran las universidades y coadyuvar al mejoramiento de la sociedad. En esta línea, el presente trabajo pretendió analizar la calidad educativa del programa de Psicología de la Corporación Universitaria Del Caribe-Cecar de Sincelejo, Sucre. Específicamente de la condición n², que alude a la justificación, y en particular la pertinencia del programa académico a partir del estado actual, tendencias y líneas de desarrollo en el ámbito internacional y nacional, la pertinencia así como el estudio de mercado.

\section{Método}

La presente investigación se enmarca en un paradigma interpretativo, el cual establece un método de investigación científica para conocer la realidad y profundizar en el conocimiento, llegando a la compresión e interpretación de los fenómenos estudiados. De acuerdo con Hernández-Sampieri, Méndez y Mendoza (2014), el trabajo se limita a un enfoque cualitativo de la investigación científica, que comprende la recolección y análisis de los datos con el fin de generar un proceso de interpretación por medio de la información esquemática. Se trata, además, de una investigación de revisión documental-descriptiva, la cual genera un conjunto de criterios de revisión necesarios para el análisis de un documento escrito y otros. 


\section{Población y muestra}

Población: Constituida por el documento maestro para la renovación del Registro Calificado del programa de Psicología de la Corporación Universitaria del Caribe CECAR.

Muestra: Condición n02 Justificación

\section{Procedimiento}

De acuerdo con los objetivos de la investigación, se empezó con la solicitud de obtención del documento maestro del registro calificado de la corporación, específicamente al personal encargado de la construcción de dicho material. Después, se identificó la condición n² Justificación del programa de Psicología. A continuación, se ubicaron los puntos clave necesarios para llevar a cabo el análisis del trabajo y, subsiguientemente, se estableció una categorización a partir de los fundamentos de la condición de justificación. Por último, se esquematizó la información y se presentó una estructuración de los hallazgos encontrados.

\section{Resultados}

Las instituciones de educación superior deben implementar sistemas de gestión de la calidad que les permitan organizar y estructurar componentes claves de mejoramiento (Vesga, 2013). A continuación, se presenta la guía de configuración, donde se encuentra la esquematización de la revisión del documento, condición n² justificación.

\section{Guía de esquematización}

En el esquema se visualizan los tres criterios clave de la Condición n02 justificación del programa de Psicología de la Corporación Universitaria del Caribe CECAR, para la obtención del Registro Calificado. El primer criterio es el estado actual, tendencias y líneas de desarrollo, en el que se esclarece un panorama acorde con los retos, la formación y los cambios. El segundo criterio es la pertinencia del programa de Psicología, donde se manifiesta el estado actual de la salud mental y la contextualización desde un nivel nacional y regional-local. El tercer criterio es el estudio de mercado del programa. En este último se presentan informes de graduados y de la oferta de pregrado en Psicología. 


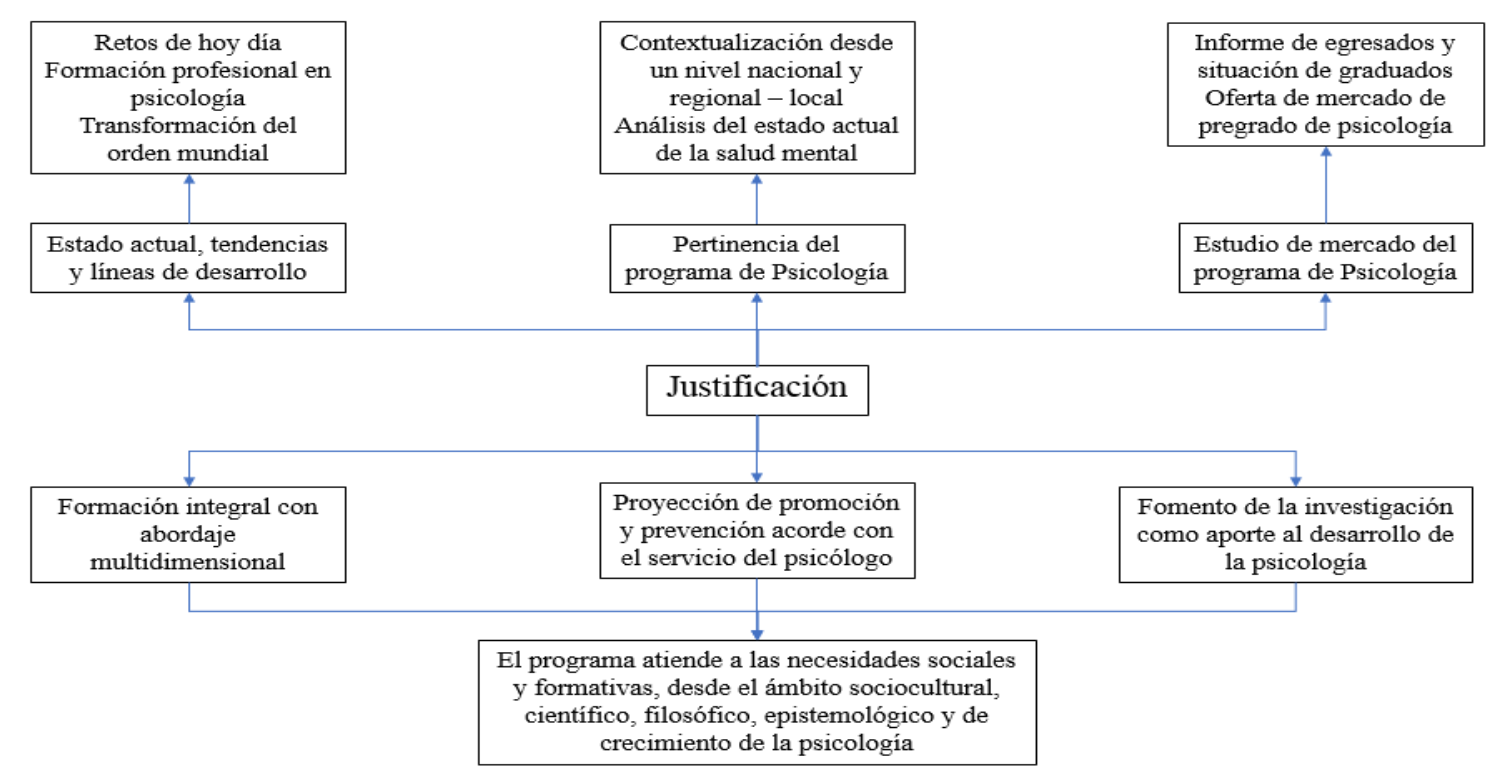

Figura 1. Esquematización de criterios de análisis. Fuente: Elaboración propia (2019).

Las tendencias generales de la educación superior en Colombia pretenden definir los caminos hacia los que se está proyectando en el futuro, los cuales son similares en todas las naciones del mundo. Sin embargo, la dimensión social, económica y política son factores que inciden directamente en las necesidades y en el progreso de la educación (Restrepo, 2006). En lo que concierne al primer criterio de estado actual, tendencias y líneas de desarrollo, se realiza un análisis desde el contexto internacional y nacional. En el primero, se descubren aportes desde distintas regiones, lo que permite inferir que se llevó a cabo una búsqueda exhaustiva en torno al impulso y tendencias en Psicología. En este contexto, CECAR comparte semejanzas y diferencias con los aportes buscados. Por otro lado, el programa de Psicología es coherente con los modelos europeos y estadounidenses. A nivel nacional, se encuentran aportes de autores en relación con la organización académica y se entiende la Psicología como una profesión y no a modo de una disciplina y de currículo, haciendo énfasis en la formación moderna y la globalización. Se trata de un fenómeno general, donde se debe establecer una fundamentación basada en competencias. En el caso de la Corporación CECAR, se señalan algunas necesidades en relación con los métodos de enseñanza y de evaluación acorde con las exigencias de la sociedad colombiana. 


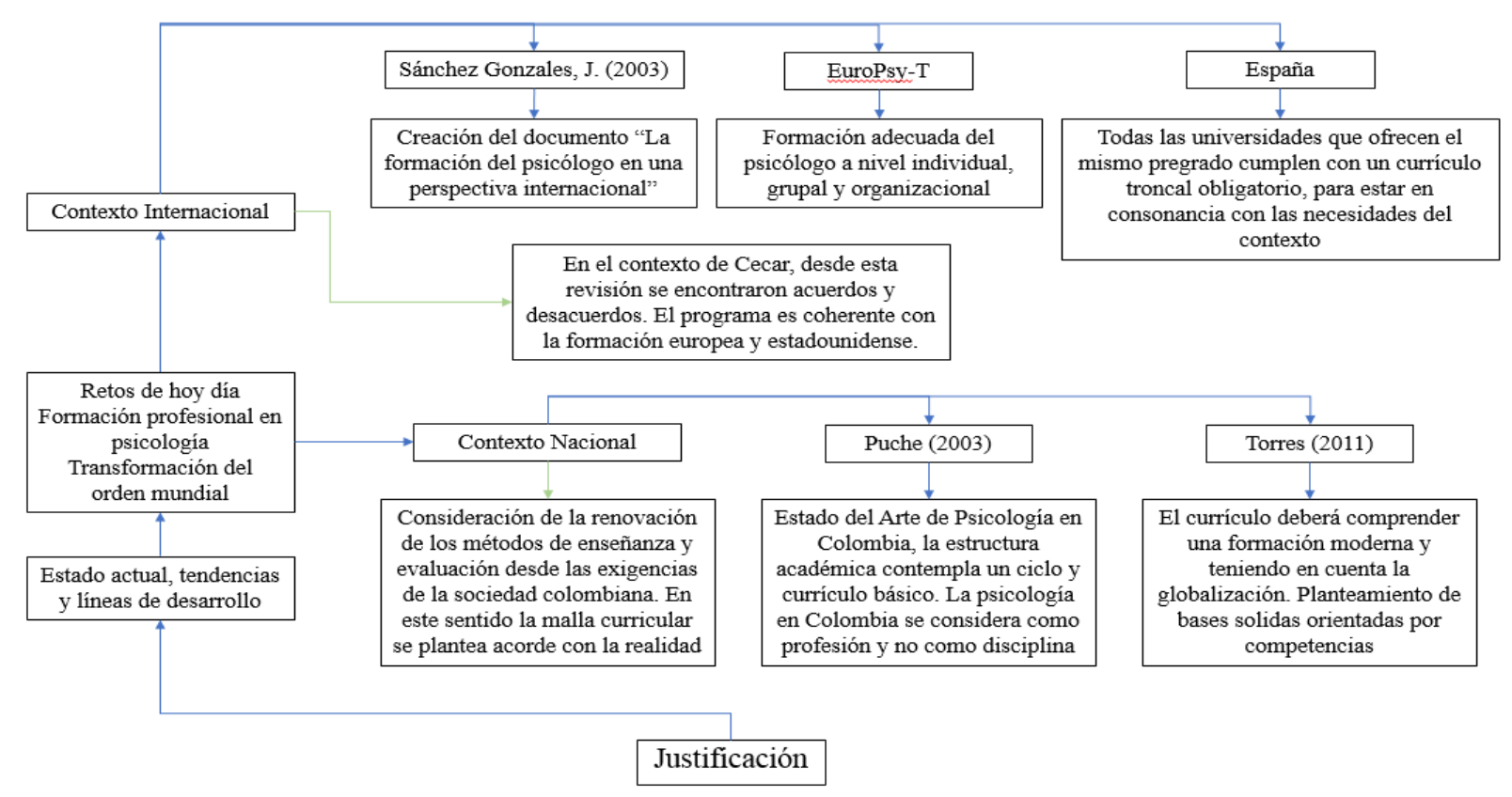

Figura 2. Estado actual, tendencias y líneas de desarrollo. Fuente: Elaboración propia (2019).

El logro de la calidad de la educación se centra en la definición de objetivos claros y alcanzables, que visibilicen la consecución de los retos de la actualidad y del gobierno nacional, adecuando los modelos de evaluación basados en las competencias y garantizando estándares básicos para la excelencia educativa (Echavarría \& Vasco, 2006). En cuanto a la pertinencia del programa de Psicología a partir del contexto nacional y regional-local, se rescatan, en primer lugar, aportes que permiten esclarecer situaciones críticas en las que se encuentra nuestra sociedad en relación con la salud mental $y$, al mismo tiempo, se identifican las oportunidades. En este sentido, a nivel territorial, existen muchas deficiencias en el entorno colectivo. En segundo lugar, se encontraron preocupantes fenómenos de salud pública en el ambiente comunitario, como NBI, pobreza, homicidios, masacres, por lo cual se confirma la necesidad de formar profesionales en las Ciencias Sociales Humanas, puntualizando en expertos psicólogos con las capacidades necesarias para obtener cambios en la comunidad. (Peña-Correal, 2007). En este marco, desde los años 70 se tomó conciencia acerca de la profesión y se dio paso a una nueva era, basada en la congruencia social y la investigación. 


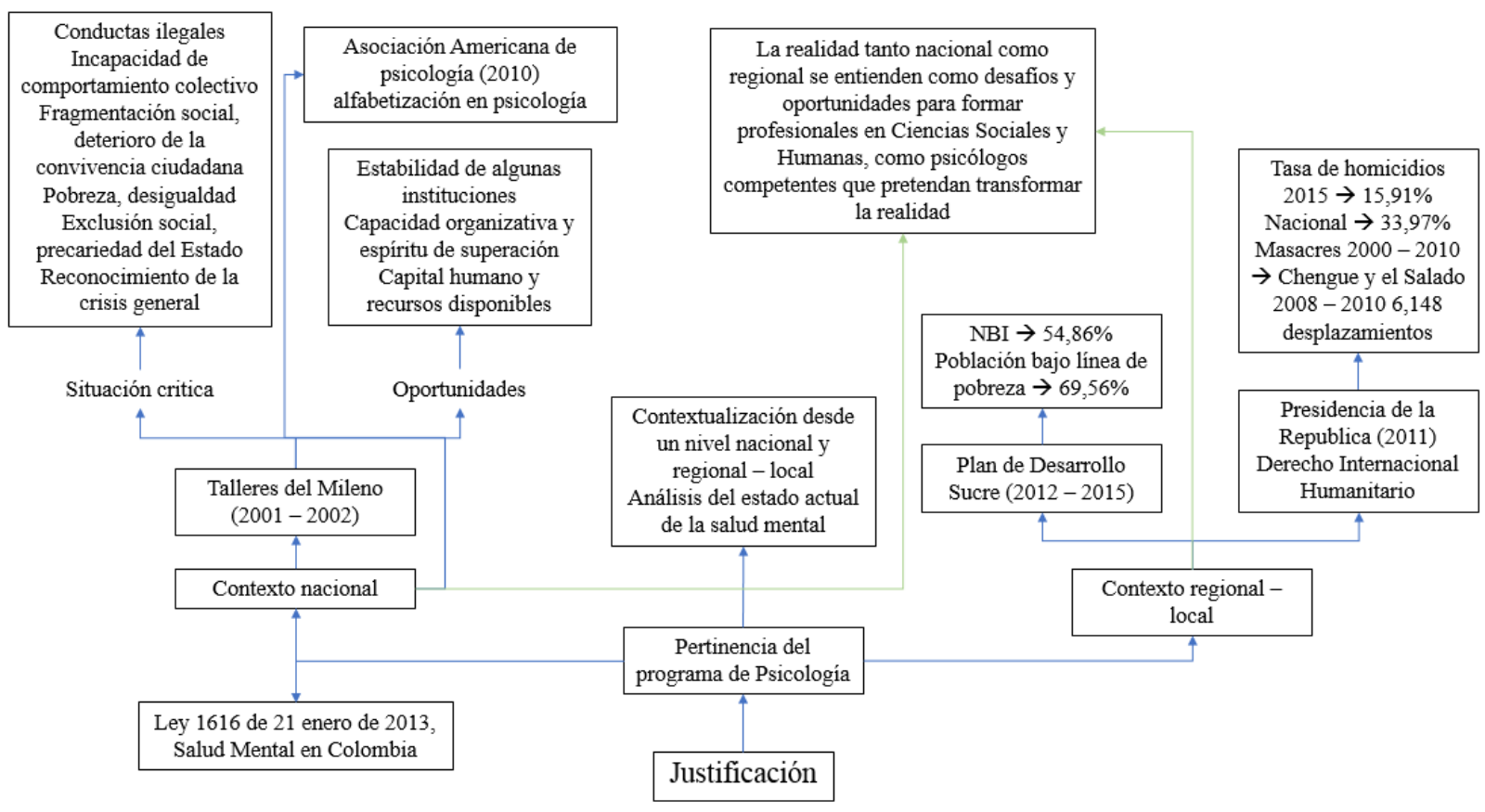

Figura 3. Pertinencia del programa de psicología. Fuente: Elaboración propia (2019).

Ardila (1967) comenta que tras la instauración de la Psicología en Colombia, solo la Universidad Nacional y la Javeriana ofrecían este programa profesional, cuyas 12 primeras promociones se basaron en materias básicas, humanidades, biología y matemáticas. Así mismo, Silva (2007), haciendo un acercamiento a los cambios de la disciplina, destaca aquellos relacionados con la formación social, neurológica y conductual. Argumenta este autor que esta ocupación es predominante, con más de 100 programas de pregrado, representando autonomía y demostrándolo en los diferentes campos donde los egresados se desempeñan. Por otra parte, Rama (2006) argumenta que las reformas realizadas a los sistemas educativos se pueden entender como un monopolio a cargo del Estado, específicamente ligados al sector político y económico del país. En este sentido, la estabilidad o el desequilibrio del mercado nacional repercuten en el área educativa. Escorcia, Gutíerrez y Henríquez (2007) aseveran que las demandas en los principales contextos de la nación requiere profesionales que posean capacidad para demostrar las habilidades y competencias que adquirieron en las universidades, entendiendo que en lo pertinente a la educación superior, en Colombia se valora desde la elección de una carrera universitaria hasta la graduación, y se adquiere una mayor rentabilidad de ese recurso humano (Hernández, 2010). En cuanto a la interpretación de mercado del programa de Psicología de la CECAR, se hace un análisis de la situación de los estudiantes titulados y de la concurrencia académica de pregrado en esta misma. Se encontró información acorde con las tasas de egresados en un trayecto de tiempo y los datos establecen una tendencia inclusive en años actuales. También aparecen estadísticas de graduados en un periodo de 2001 a 2009. La oferta educativa en distintas ciudades y departamentos (Bogotá, Antioquia, 
Valle y Atlántico) indica que existe una necesidad clara de formar psicólogos en el Caribe colombiano. Actualmente, existen 106 programas distribuidos en 85 instituciones de instrucción superior, en 42 municipios del país. De estos, 83 tienen registro calificado, pero solo 21 cumplen con la acreditación de alta calidad y entre esos se encuentra el programa de Psicología de la Corporación Universitaria del Caribe CECAR de la ciudad de Sincelejo.

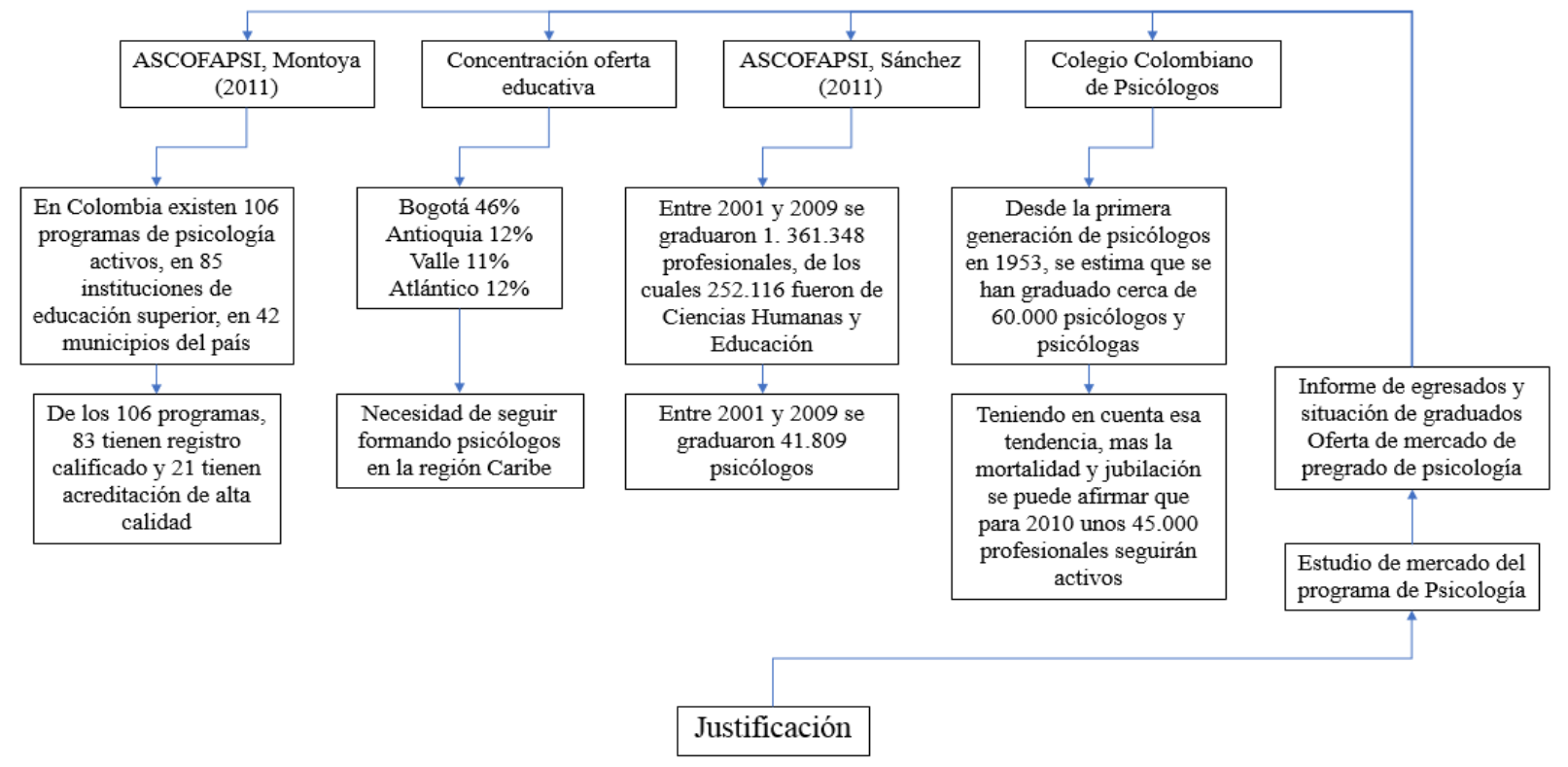

Figura 4. Estudio de mercado del programa de psicología. Fuente: Elaboración propia (2019).

\section{Discusión}

Las principales falencias que impiden que las universidades obtengan las acreditaciones, se deben a que, en general, presentan modelos basados en el sistema administrativo y desestiman la importancia de la institución. En estas circunstancias, los procesos de acreditación funcionan como una fachada, presentando poca participación de la comunidad académica, un régimen de evaluación centrado en los contenidos y no en las competencias y falta de visión al seguimiento de acreditar, entendiéndolos como un paso fundamental para la transformación de la educación y de la sociedad (Martínez, Tobón \& Romero, 2017). Castaño-Duque y García (2012) corroboran lo anterior y argumentan que, a causa de todos los inconvenientes que se han presentado alrededor del aprendizaje, se creó el procedimiento de aseguramiento de la calidad, encargado de llevar los métodos institucionales que posibilitan la obtención de certificación o notificación de alta eficacia. A su vez, Misas (2004) sostiene que las metodologías educacionales hacen parte de los planes de desarrollo económico, social y político de una nación; por tanto, las instituciones y centros que proporcionan servicios de enseñanza deben mantener un compromiso en la capacitación de profesionales aptos para enfrentar conveniente y comprometidamente el progreso de la humanidad. Mientras que Orozco (2010) plantea las dificultades del adiestramiento 
universitario ante los efectos de la globalización y de una creciente reestructuración de los mercados laborales, que generan muchos cambios en poco tiempo y, por ende, las entidades deben atravesar etapas de adaptación muy rápidas, estableciendo estándares dudosos con el fin de "cumplir" las demandas. (Rico, 2016). Asevera también que la gestión de los establecimientos de formación superior debe asegurar ciertos criterios que incluyan una mayor relevancia en la continuidad educativa, bien sean programas de investigación o sistemas de preparación del personal y cuerpo docente.

De acuerdo con Ruiz, Jaraba y Romero (2008), el área de psicología demanda requerimientos obligatorios para identificar la calidad de los egresados con relación a conocimientos, habilidades, capacidades, actitudes y destrezas, como condiciones que proporcionen un excelente desempeño en el ámbito laboral. Ello a diferencia de Peña (2013), para quien no se debe esperar falencias de los profesionales de la salud mental cuando la institución cuente con programas que evalúen el avance del educando y de los modelos educativos que se implementan, atendiendo a su carácter multiparadigmático. Según Facundo (2003), los establecimientos de educación superior deben elaborar planes estratégicos y remodelados que, conforme a las demandas y avances del mundo, reorienten hacia la transformación comunitaria. Por otra parte, para Rama (2006) la enseñanza se ha convertido en un bien comercializado, el cual es protegido por entes que solo están interesados en obtener ganancias a costas de un mal servicio educativo; y Ospina y Sanabria (2010) piensan que las ofertas académicas que se brindan hoy en día correspondan a las necesidades del sector productivo y al mismo tiempo a los de la sociedad. En una dirección parecida, Malagón (2003) advierte que los sistemas pedagógicos requieren una sintonía con el orden mundial actual, y su dinamismo, interconectado con la sección productora y social, forjando así integralidad. Fontalvo, Mendoza y Morelo (2011) explican, a su tiempo, que los procesos que lleva a cabo el sistema de gestión impacta directamente la rentabilidad de las empresas en forma positiva o negativa.

Ahora bien, los objetivos de la investigación se encaminaron hacia el análisis de tres (3) aspectos básicos de la condición n02 del registro calificado del pregrado de Psicología de la Corporación Universitaria del Caribe, los cuales se desarrollaron de manera efectiva y eficaz, al encontrar lo que se requiere para la solidificación de un plan acreditado en alta calidad. El objetivo número uno buscó, en efecto, examinar las tendencias y líneas de desarrollo. Las observaciones indican que se hizo una caracterización de las predisposiciones y estilos de progreso, a partir de dos niveles, internacional y nacional. Seguidamente, se realizó una comparación con lo encontrado y lo implementado en la institución. Así, en el primer nivel se identificaron semejanzas entre los modelos implementados, acordes con el europeo y el estadounidense. Aquí se asume un nivel neutro de relevancia, acorde con lo que menciona Peiró (2003) en relación con el crecimiento del área de psicología según las demandas que el medio exige y manifiesta. De este modo, con el paso de los años, los cambios han traído consigo aspectos significativos para esta ciencia, evolucionando considerablemente para atender las preocupaciones y buscar el bienestar de la sociedad. En el caso de Europa, se ha consolidado un ambiente para la formación en psicología (enseñanza-aprendizaje), 
partiendo de exploraciones y nuevos esquemas de formación, principalmente la basada en competencias, concretándose dentro de estos planes un requerimiento máximo a la hora de acreditar títulos. Es claro que este continente lleva una enorme delantera, con un alto índice de desarrollo en el modelo educativo de la Psicología. Rey, Martínez y Guerrero (2009) confirman, en tal sentido, que España es uno de los países donde más surgen artículos investigativos, lo que explica que los países americanos tiendan a asemejar sus modelos con los europeos.

En el segundo apartado, se identifica una renovación de los métodos de enseñanza con el fin de promover la exigencia. La meta dos investigó la pertinencia de la carrera, para lo cual se realizó una contextualización en todo el país y de tipo regional-local, identificándose las necesidades sociales de la sociedad. Esto último incluye desde problemáticas psicosociales hasta oportunidades que permitan una buena apropiación del programa en el contexto en el que se encuentra ubicado. Según Posada (2013), en Colombia poco menos de la mitad de la población es proclive a sufrir por lo menos alguna vez en su vida un trastorno mental psiquiátrico, entre los 18 y 65 años de edad. La depresión es uno de los más comunes, así como los trastornos relacionados con el estado del ánimo, control de impulsos y consumo de sustancias psicoactivas; el autor asume que en la presente década se deben tomar acciones relacionadas con la prevención de la enfermedad mental en el país, proponiendo metas de desarrollo con la calidad de la sociedad, de manera que la educación en el área psicológica y semejantes constituyen un gran potencial para alcanzar esos propósitos.

El tercer y último alcance indagó el estudio de mercado, demostrando que en Colombia y en el Caribe existe una gran demanda de profesionales formados en las ciencias humanas. Porcentualmente los espacios del país que requieren psicólogos comprenden: Bogotá, con un $46 \%$, Antioquia, $12 \%$, Valle, $11 \%$ y Atlántico, $12 \%$. De igual modo, como ya se ha dicho, solo 21 programas de los 106 existentes están acreditados con un grado de excelencia significativo, y entre estos CECAR ocupa un puesto privilegiado. La Corporación Universitaria del Caribe lleva 32 años educando en la región Caribe, con un total de 30.362 graduados desde entonces. Una reciente participación en el Taller de Formación de Pares Evaluadores llevado a cabo en la Universidad Simón Bolívar, reconoció a CECAR con la implementación de indicadores en sus programas de pregrado tanto regional, nacional como internacionalmente (Corporación Universitaria del Caribe CECAR, 2019).

Los hallazgos encontrados demuestran el grado de importancia que imparte la institución a la calidad de la educación, ofreciendo un servicio acorde con las necesidades del medio y apropiado para la comunidad académica en general. Las limitaciones, en este sentido, apuntan hacia un análisis extenso de todos los aspectos que se evalúan en la justificación. Sin embargo, el fin del trabajo fue centrarse en las características más relevantes, acordes con el estado de la sociedad donde se ofrece. Se resalta que uno de los puntos fuertes del estudio es la demostración abierta de la investigación como una división específica del documento maestro del programa de pregrado. 


\section{Conclusión}

La calidad de la educación en Colombia presenta déficits relacionados con la cobertura, pertinencia, eficacia y eficiencia. El gobierno central no ha tenido la capacidad para dar respuestas y alternativas de solución a estos problemas que afectan las acciones académicas, en los centros de todos los niveles educativos. Según Aguila (2005) esto es debido a que no existe relación entre lo que refiere la ley y lo que practican las instituciones. Muchos críticos y estudiosos de la comunidad académica y científica plantean que la no resolución de los inconvenientes en el marco educacional conlleva una debilidad del Estado Nacional y obstaculiza el avance y progreso de la sociedad (Ardila-Rodríguez, 2011). Coincidiendo con lo anterior, Ayala (2010) advierte que la denominada Revolución Educativa es una mal llamada propuesta enfocada en propósitos provechosos para el sistema político, pero en caso de llegarse a cumplir se obtendrá un nuevo nivel de formación superior, pues, el medio educativo se ve influenciado por las demás entidades sociales y el modelo económico. En este sentido, como afirma García (2005), la comercialización y la globalización han impactado la academia de forma significativa, a causa de que se vio incluida en los servicios que son negociados por la administración del Estado. Y es que este bien es indispensable en su productividad, es decir, genera crecimiento a partir de la formación en la que se fundamenta, expresados mediante el desarrollo y progreso de una nación o sociedad en términos de profesionalización y empleo (Eicher, 1988).

El programa de Psicología de la Corporación Universitaria del Caribe CECAR se halla entre los pocos programas del país acreditados con alta calidad. La justificación como condición n02 para la eficacia, ubicada en el documento maestro enfatiza de forma clara y profunda en los criterios básicos y contempla la pertinencia, ahondando en contextos internacionales y nacionales, lo que permite establecer grados de comparación situacional. Sin embargo, según Torres (2011), el psicólogo colombiano necesita formarse en un modelo educativo basado en competencias que definan la profesionalización del perfil acorde con las necesidades del servicio; lo que representa un déficit entre la relación de lo que se requiere y lo que se tiene. De manera concreta, en la región Caribe existen insuficiencias en materia de salud mental que deben ser satisfechas, y ello implica formar profesionales integrales, que provean un nuevo sentido de transformación y cambio para nuestra sociedad. Por esta razón, la educación que los psicólogos reciban debe ser óptima y multidimensional. Uribe (2013) afirma que existen muy buenas percepciones acerca de los modelos de acreditación que utilizan las universidades colombianas para acceder a la excelencia, pero de todos modos hay una crítica externa particular sobre la extensión y no contextualización de estos. Marín-Agudelo y Carrasco-Tapias (2012) añaden que los planes regionales de psicología se deberían asemejar a los procedimientos y sistemas mundiales, teniendo en cuenta que el factor de la globalización y las tendencias actuales en dicho pregrado faciliten el crecimiento de la profesión.

Para Alzate-Medina (2008), los programas de Psicología que tienen la necesidad de 
acreditarse representan una dificultad para los procesos de mejoramiento y autorregulación en los aspectos relacionados con el fomento de la importancia de la calidad en la comunidad académica. El trabajo comprende una visión analítica desde una esquematización en el que se localizaron los aspectos más relevantes de la información relacionada con la justificación como condición mínima de la calidad. El crecimiento de un programa de pregrado debe incluir el esfuerzo por convertirse en una institución líder, y el programa de Psicología de CECAR ha demostrado, dentro de su proceso de evolución, que se puede distinguir en la costa Caribe como una institución que forma para y por el futuro. Sin embargo, aún no se puede establecer un grado de comparación con las grandes universidades del país, debido a que existen brechas notorias, desafíos que deben ser superados y retos por alcanzar. No cabe duda, en fin, que la institución puede, en un determinado tiempo, convertirse en una de las mejores universidades de Caribe colombiano. Como se sabe, la calidad es un proceso continuo al cual se compromete una institución, la cual debe alinearse a los parámetros y criterios que administran el sistema educativo en Colombia (Londoño \& Ramírez, 2012) o, en otras palabras, comprometerse con los objetivos, integralidad, coherencia y responsabilidad que sobrellevan la calidad. Guerrero (2004) sostiene que en la actualidad la educación superior comprende retos diarios, particularmente el de la globalización, por lo que esta se tiene que reconfigurar cada cierto tiempo para ser útil como entidad productora de conocimiento, ante las exigencias del futuro y de la competencia. Duque y Chaparro (2017) coinciden en lo anterior, argumentando que la educación no puede permanecer en un estado de inmovilización, a riesgo de atrofiarse y perderse, sino que debe mantenerse activa, fulgurante, continua en el desarrollo y en la formación de excelentes profesionales. Por tanto, la educación debe asumir los retos por venir, lo que incluye el compromiso de reorganizar e implementar un sistema de evaluación de la calidad autónomo e independiente que sobrelleve los cambios de las prácticas pedagógicas y le evolución de procesos que beneficien el entorno local y nacional (López, 2007). Robledo (2008) comenta que el contexto colombiano cada vez demanda más psicólogos expertos para atender las problemáticas sociales y así mantener la pertinencia de la educación desde el ámbito científico y pedagógico. Tradicionalmente, las universidades e instituciones de educación son lugares donde se enseña a pensar, pero en las últimas décadas esto ha cambiado, conceptuando la educación como un medio facilitador de la formación de mano de obra (Parra \& Carvajal, 1979). Lo anterior ha generado muchas críticas en torno a las competencias laborales, entrenamiento y capacitación de los universitarios en Psicología, haciendo alusión a las exigencias diversas que suscita el mercado en general (Gómez, 2015).

Para finalizar, se espera que este documento no sea solo un acercamiento al análisis de la calidad de un programa de pregrado, sino que sea entendido como parte de una consolidación conceptual, referencial y científica acerca del sistema de educación superior en concordancia con la pertinencia de los procesos de alta calidad para el área de Psicología de la ciudad de Sincelejo y la Corporación Universitaria del Caribe. CECAR se visualizó y consolido entre las entidades de educación superior que demuestra avances en el diseño y organización de la formación universitaria a nivel regional (Corporación Universitaria del 
Caribe CECAR, 2017). Y el Sistema Integrado de Gestión de Calidad está acorde con los retos de la Corporación en relación con el cuerpo docente, índice de investigación, proyección social y reconocimiento internacional, dando como resultado el cumplimiento de las normativas para la calidad de la educación superior.

\section{Referencias}

Aguerrondo, I. (1993). La calidad de la educación: Ejes para su definición y evaluación. Revista Interamericana de desarrollo educativo, 37(116), 561-578.

Aguila, V. (2005). El concepto calidad en la educación universitaria: clave para el logro de la competitividad institucional. Revista Iberoamericana de Educación, 36(12), 1-7.

Alzate-Medina, G. (2008). Efectos de la acreditación en el mejoramiento de la calidad de los programas de psicología de Colombia. Revista Universitas Pshychologica, 7(2), 425439.

Ardila, R. (1967). La psicología en Colombia. Revista Interamericana de Psicología, 1(4), 239-249.

Ardila-Rodríguez, M. (2011). Calidad de la educación superior en Colombia, ¿Problema o compromiso colectivo? Revista Educación y Desarrollo Social, 5(5), 44-55.

ASCOFAPSI. (2012).¿Qué es el Observatorio de la Calidad de la Educación Superior en Psicología en Colombia? Observatorio de la Calidad de la Educación Superior en Psicología en Colombia, 1+. Recuperado de http://observatorio.ascofapsi.org.co/

Ayala, M. (2010). Financiamiento de la educación superior en Colombia reflexiones para un próximo futuro. Revista de la educación superior, 4(156), 89-102.

Beltrán-Villamizar, Y., Matínez-Fuentes, Y. \& Vargas-Beltrán, Á. (2015). El sistema educativo colombiano en el camino hacia la inclusión: Avances y retos. Revista Educación y Educadores, 18(1), 62-75.

Bonilla, L. (2011). Doble jornada escolar y calidad de la educación en Colombia. Coyuntura Económica, XII (1) 63-103.

Brunner, J. (2010). Educación superior en América Latina. Centro de Políticas Comparadas de Educación. Recuperado de https://www.researchgate.net/publication/313423057_Educacion_Superior_en_Ibe roamerica-_Informe_2016

Burton, C. (1991). El sistema de educación superior. Una visión comparativa de la organización académica. En Burton, C. El sistema de educación superior. Una visión comparativa de la organización académica. México: Editorial Nueva Imagen. 
Castaño-Duque, G. \& García, L. (2012). Una revisión teórica de la calidad de la educación superior en el contexto colombiano. Revista Educación y educadores, 15(2) 219-243.

Celis, M., Jiménez, A. \& Jaramillo, J. (2012). ¿Cuál es la brecha de la calidad educativa en Colombia en la educación media y superior? ICFES. Recuperado de https://www2.icfes.gov.co/documents/20143/233733/Cual+es+la+brecha+de+cali dad+educativa+en+colombia+en+educacion+media+y+superior.pdf

Colbert, V. (1999). Mejorado el acceso y la calidad de la educación para el sector ruralpobre. El caso de la Escuela Nueva en Colombia. Revista Iberoamericana de Educación, (20), 107-135.

Congreso de Colombia. (8 de febrero de 1994).Ley General de Educación. [Ley 115 de 1994]. DO: 41.214.

Consejo Nacional de Acreditación CNA. (1992). Ley 30 de diciembre 28 de 1992. Bogotá: Congreso de Colombia. Recuperado de https://www.cna.gov.co/1741/articles186370_ley_3092.pdf

Corporación Universitaria del Caribe CECAR. (2017). Avances y direccionamiento de la educación superior a nivel regional. Recuperado de https://cecar.edu.co/documentos/dian/2-informe-de-gestion.pdf

Corporación Universitaria del Caribe CECAR. (2019). CECAR, a la vanguardia de la calidad educativa en Latinoamérica. Recuperado de https://cecar.edu.co/documentos/dian/2-informe-de-gestion.pdf

Duque, E. \& Chaparro, C. (2017). Medición de la percepción de la calidad del servicio de educación por parte de los estudiantes de la UPTC Duitama. Revista Criterio Libre, 10(16), 159-192.

Echavarría, C. \& Vasco, E. (2006). Justificaciones morales de lo bueno y lo mal en un grupo de niñas y niños provenientes de contextos violentos y no violentos de una ciudad de la región andina de Colombia. Acta Colombiana de Psicología, 9(1), 51-62.

Eicher, J. (1988). Treinta años de economía de la educación. Ekonomiaz: Revista vasca de economía, (12), 11-38.

Escorcia, R., Gutiérrez, A. \& Henríquez, H. (2007). La educación superior frente a las tendencias sociales del contexto. Revista de Educación y educadores, 10(1), 63-77.

Facundo, Á. (2003). La educación superior virtual en Colombia. La educación superior virtual en América Latina y el Caribe. Recuperado de https://unesdoc.unesco.org/ark:/48223/pf0000139922

Fontalvo, T., Mendoza, A. \& Morelo, J. (2011). Evaluación del impacto de los sistemas de gestión de la calidad en la liquidez y rentabilidad de las empresas de la Zona Industrial de Mamonal (Cartagena - Colombia). Revista Virtual Universidad Católica del Norte, (34), 314-341. 
Garbanzo, G. (2007). Factores asociados al rendimiento académico en estudiantes universitarios, una reflexión dese la calidad de la educación superior pública. Revista Educación, 31(1), 43-63.

García, C. (2005). Complejidades de la globalización e internacionalización de la educación superior. Revista Cuadernos del Cendes, 22(58), 1-22. Recuperado de https://www.redalyc.org/pdf/403/40305802.pdf

Giraldo, U., Abad, D. \& Díaz, E. (2007). Bases para una política de calidad de la educación superior en Colombia. Recuperado de https://redes.colombiaaprende.edu.co/ntg/men/pdf/Lineamientos.pdf

Gómez, M. (2015). El sentido de la formación universitaria en psicología. Revista de Investigaciones, 15(25), 112-128.

Gómez, V. \& Celis, J. (2009). Crédito educativo, acciones afirmativas y equidad social en la educación superior en Colombia. Revista de Estudios Sociales, (33), 106-117.

Guerrero, G. (2004). La educación en el contexto de la globalización. Revista Historia de la Educación, 6(6), 343-354. Recuperado de https://www.redalyc.org/pdf/869/86900620.pdf

Hernández, G. (2010). ¿Cuán rentable es la educación superior en Colombia? Revista Lecturas de Economía, (73), 181-214.

Hernández-Sampieri, R., Méndez, S. \& Mendoza, C. (2014). Metodología de la investigación. En R. Hernández Sampieri, Metodología de la investigación, sexta edición. México: McGraw-Hill - Interamericana Editores, S. A. de C. V

Herrera, A., Restrepo, M., Uribe, A. \& López, C. (2009). Competencias académicas y profesionales del psicólogo. Revista Diversitas: Perspectivas en Psicología, 5(2), 241254. Recuperado de https://www.redalyc.org/pdf/679/67916260004.pdf

Londoño, J. \& Ramírez, D. (2012). Cultura de la autoevaluación: cimiento para la acreditación de programas académicos en modalidad virtual. Revista Virtual Universidad Católica del Norte, 1(36), 1-6. Doi: 10.35575/rvucn.n36a1

López, N. (2007). Acerca de la problemática de la evaluación de la calidad de la educación en Colombia. Revista Latinoamericana de Estudios Educativos, 3(2), 9-28.

Malagón, L. (2003). La pertinencia en la educaicón superior. Elementos para su compresión. Revista de Educación Superior, XXXII (3), 1-25.

Marín-Agudelo, H., \& Carrasco.Tapias, N. (2012). Análisis comparativo de la pertinencia del programa de psicología de la Universidad Cooperativa de Colombia a la luz del contexto nacional e internacional de los programas de psicología. Revista Pensando Psicología, 8(14), 59-81.

Martínez, J., Tobón, S. \& Romero, A. (2017). Problemáticas relacionadas con la acreditacón de la calidad de la educación superior en América Latina. Revista Innovación 
Educativa, (17), 79-96.

Melo-Becerra, L., Ramos-Forero, J. \& Hernández-Santamaría, P. (2017). La educación superior en Colombia: situación actual y análisis de eficiencia. Revista de Desarrollo y Sociedad, (1), 59-111.

Ministerio de Justicia. (2009). Decreto 2566 de 2003. Recuperado de http://www.suinjuriscol.gov.co/viewDocument.asp?id=1771011

Miñana, C. \& Rodríguez, J. (2011). Calidad, evaluación y acreditación en la educación superior: a propósito de un proyecto de reforma en Colombia. Revista Pensamiento jurídico, (31), 155-185.

Misas, G. (2004). La educación superior en Colombia: análisis y estrategias para su desarrollo. En Misas, G. La educación superior en Colombia: análisis y estrategias para su desarrollo. Bogotá: Universidad Nacional de Colombia, UNIBIBLOS.

Naranjo, M. (2009). Motivación: perspectivas teóricas y algunas consideraciones de su importancia en el ámbito educativo. Revista Educación, 33(2), 153-170.

Orozco, L. (2010). Calidad académica y relevancia social de la educación superior en América Latina. Revista iberoamericana de educación superior, 1(1), 24-36.

Ospina, M. \& Sanabria, P. (2010). Un enfoque de mercadeo de servicios educativos para la gestión de las organizaciones de educación superior en Colombia: el modelo migme. Revista Facultad de Ciencias Económicas, XVIII (2) ,107-136.

Parra, R. \& Carvajal, M. (1979). La universidad colombiana: de la filosofía a la tecnocracia estratificada. Revista Colombiana de Educación, (4), 1-11.

Peiró, J. (2003). La enseñanza de la psicología en Europa. Un proyecto de titulación europea. Papeles del Psicólogo, 24(86), 25-33.

Peña-Correal, T. (2007). 60 años de la Psicología en Colombia. Revista Latinoamericana de Psicología, 39(3), 675-676.

Peña, J. (2013). La psicología como disciplina científica multiparadigmática: planteamientos preliminares para fundamentar un programa educativo profesional. Revista Integración Académica en Psicología, 1(1), 22-97.

Peralta, B. (2009). La formación ciudadana en el sistema educativo de Colombia cuna mirada reactiva o transformadora? Revista Eleuthera, (3) ,165-178.

Posada, J. (2013). La salud mental en Colombia. Biomédica, 33(4), 497-498.

Rama, C. (2006). La tercera reforma de la educación superior en América Latina y el Caribe: masificación, regulaciones e internacionalización. Revista de Educación y Pedagogía, $18(46), 11-24$.

Rama, G. (1970). El sistema universitario en Colombia. Revista de la Universidad Nacional, (6), 188-209. 
Restrepo, B. (2006). Tendencias actuales en la educaicón superior: rumbos del mundo y rumbos del país. Revista Educación y Pedagogía, XVIII (46), 79-90.

Restrepo, M. (2003). Necesidades reales de formación de psicólogos en el país y en la región. Poiesis, (6), 1-5.

Rey, C., Martínez, J. \& Guerrero, S. (2009). Tendencias de los artículos en psicología clínica en Iberoamérica. Revista Terapia Psicológica, 27(1), 61-71.

Rico, A. (2016). Hacía la optimización de la formación docente en la educación superior en Colombia. Revista Sophia, 12(1), 55-70.

Roa, A. (2003). Hacia un modelo de aseguramiento de la calidad en la educación superior en Colombia: estándares básicos y acreditación de excelencia. Recuperado de https://www.mineducacion.gov.co/1621/article-87155.html

Robledo, Á. (2008). La formación de psicólogos y psicólogas en Colombia. Revista Universitas Psychologica, 7(1), 9-18.

Ruiz, M., Jaraba, B. \& Romero, L. (2008). La formación en psicología y las nuevas exigencias del mundo laboral: Competenias laborales exigidas a los psicólogos. Revista Psicología desde el Caribe, (21), 136-142.

Salas, W. (2005). Formación por competencias en educación superior. Una aproximación conceptual a propósito del caso Colombiano. Revista Iberoamericana de Educación, 36(9), 1-11.

Secretaria de Planeación. (2016 - 2019). Plan de Desarrollo del Municpio de Sincelejo 2016 - $2019 . \quad$ Sincelejo. Recuperado de http://www.alcaldiadesincelejo.gov.co/Ciudadanos/ProyectosNormatividad/Plan\%2 0de\%20Desarrollo\%20Municipal\%202016\%20-\%202019.pdf

Silva, M. (2007). La enseñanza de la psicología en Colombia. El legado rodriguista. Cambios y prospectivas. Psicogente, 10(17), 60-73.

Silva, M. \& Acevedo, Á. (2011). Formación de psicólogos y pensamiento psicológico en el Caribe colombiano 1975-2007. Cartagena, Bolívar: Universidad de Cartagena.

Tobón, S. (2006). Competencias, calidad y educación. En Tobón, S., Rial, A., Carretero, M. \& García, J. Competencias, calidad y educación. Bogotá: Cooperativa Editorial Magisterio.

Tomasevski, K. (2004). Indicadores del derecho a la educación. Revista iidh, (40), 341-388.

Torres, G. (2011). Más allá de la evaluación de las competencias del psicólogo colombiano, necesidad de un modelo de formación básico y común a nivel nacional. Revista Psychología: Avances de la Disciplina, 5(2), 121-133.

Uribe, A. (2013). Los factores que conforman el modelo de acreditación por alta calidad de programas académicos en Colombia, revisión desde el enfoque de la percepción. Revista Desarrollo Gerencial, 5(2), 21-54. 
Valencia, G. (2008). La eficacia escolar: retos y desafíos para mejorar la calidad y la equidad en el sistema educativo colombiano. Revista Uni-pluriversidad, 8(2), 23-33.

Vesga, J. (2013). Cultura organizacional y sistemas de gestión de la calidad: una relación clave en la gestión de las instituciones de educación superior. Revista Guillermo de Ockham, 11(2), 89-100.

Viloria, J. (2006). Educación superior en el Caribe Colombiano: análisis de cobertura y calidad. Recuperado

de http://www.banrep.org/docum/Lectura_finanzas/pdf/DTSER-69_(VE).pdf 